\title{
Pharmacokinetic Study of Epinephrine Hydrofluoroalkane (Primatene MIST) Metered-Dose Inhaler
}

\author{
Edward M. Kerwin, MD, ${ }^{1}$ Tony Marrs, MPH, ${ }^{2}$ Mary Z. Luo, $\mathrm{PhD},{ }^{2}$ and Jack Y. Zhang, $\mathrm{PhD}^{2}$
}

\begin{abstract}
Background: Primatene ${ }^{\circledR}$ MIST CFC, an epinephrine metered-dose inhaler (MDI), was discontinued from the market owing to environmental concerns from its use of chlorofluorocarbon (CFC) propellant. As a result, a new epinephrine MDI was developed using hydrofluoroalkane (HFA) propellant. This article reports the pharmacokinetic (PK) profile of the newly Food and Drug Administration-approved epinephrine HFA MDI. Methods: A randomized, evaluator-blinded, active-controlled, single-dose, two-arm crossover study was conducted to evaluate the PK profile of epinephrine HFA (Primatene ${ }^{\circledR}$ MIST) and epinephrine CFC (Primatene ${ }^{\circledR}$ MIST CFC) in 23 healthy volunteers to characterize the epinephrine absorption extent and rate. The study was performed at a high dose of five times the normal dose to obtain measurable plasma epinephrine levels. Plasma epinephrine levels were measured and safety was assessed by adverse events (AEs), vital signs, clinical laboratory tests, and physical examinations.

Results: Epinephrine HFA demonstrated a greater systemic drug exposure (greater area under the curve) than that of epinephrine CFC ( $\sim 37 \%$ higher). The $C_{\max }$ occurred at $\sim 2$ minutes and was significantly higher in the epinephrine HFA group $(0.18 \mathrm{ng} / \mathrm{mL})$ compared with the CFC version $(0.046 \mathrm{ng} / \mathrm{mL})$ at normal dose. Within 20 minutes, both groups demonstrated comparable plasma epinephrine levels. No clinically significant adverse effects were found to be associated with epinephrine HFA, even after an ultrahigh dose (i.e., 10 inhalations). Conclusions: The systemic exposure of epinephrine HFA was found to be higher for the first 20 minutes, and then comparable with epinephrine CFC. Minimal AEs were found in this study despite the very high 1250$2200 \mu \mathrm{g}$ inhaled doses (i.e., 10 inhalations) used for PK characterization.
\end{abstract}

Keywords: asthma, high dose, inhaled epinephrine, pharmacokinetic, systemic exposure

\section{Introduction}

$\mathbf{F}$ OR SeVeral Years, Primatene ${ }^{\circledR}$ MIST CFC was the only over-the-counter (OTC) asthma epinephrine metereddose inhaler (MDI) in the United States. Having been approved by the Food and Drug Administration in 1956, this product served a vital and unique role in meeting the OTC needs of asthma patients. However, Primatene ${ }^{\circledR}$ MIST CFC was phased out in December 2011 by the Montreal Protocol because of environmental concerns from its chlorofluorocarbon (CFC) propellant. ${ }^{(1)}$ In response, a new epinephrine MDI (Primatene ${ }^{\circledR}$ MIST) was developed that uses hydrofluoroalkane (HFA) propellant in place of CFC.

In addition to using different propellant, several other changes were made to the new formulation of Primatene ${ }^{\circledR}$ MIST. Unlike the previous CFC version, which was a solution, Primatene ${ }^{\circledR}$ MIST was formulated as a suspension. The new formulation provides enhanced drug delivery efficiency, ${ }^{(2)}$ allowing therapeutic efficacy to be achieved at a lower dose. The dose for Primatene ${ }^{\circledR}$ MIST was reduced by $43 \%$, from $220 \mu \mathrm{g} /$ inhalation in the previous $\mathrm{CFC}$ version, to $125 \mu \mathrm{g} /$ inhalation, with comparable efficacy.

\footnotetext{
${ }^{1}$ Clinical Research Institute of Southern Oregon, Medford, Oregon, USA.

${ }^{2}$ Amphastar Pharmaceuticals, Inc., Rancho Cucamonga, California, USA.

Clinical trials registration number: NCT01188577.
}

(C) Edward M. Kerwin, et al., 2020. Published by Mary Ann Liebert, Inc. This Open Access article is distributed under the terms of the Creative Commons License (http://creativecommons.org/licenses/by/4.0), which permits unrestricted use, distribution, and reproduction in any medium, provided the original work is properly credited. 
At present, very few literatures were found clearly describing the pharmacokinetic (PK) profile of epinephrine MDIs. Because concerns exist surrounding the use of epinephrine as an asthma medication, ${ }^{(3)}$ the PK profile of the new epinephrine HFA MDI was explored in a PK clinical trial.

This article reports PK systemic exposure levels of high inhaled doses of the newly formulated Primatene ${ }^{\circledR}$ MIST (epinephrine HFA, $10 \times 125 \mu \mathrm{g}$ ) and adverse events (AEs) reported during the trial. The results are compared with an active control product, Primatene ${ }^{\circledR}$ MIST CFC $(10 \times 220 \mu \mathrm{g})$.

\section{Materials and Methods}

\section{Study design}

This randomized, evaluator-blinded, active-controlled, single dose, two-arm crossover PK study in healthy subjects was conducted at a single site located in the United States. Because doses administered through inhalation may be too small for detection in plasma concentration, ${ }^{(4)}$ a high dose of five times the normal dose (i.e., 10 inhalations) was used to ensure adequate measurements of plasma concentrations for PK analysis. To thoroughly explore the PK profile of Epi-HFA, a stable isotope deuterium-labeled epinephrine was used to differentiate the administered exogenous epinephrine (epinephrine-d3) from the endogenous epinephrine (epinephrine-h3).

The study consisted of a screening visit and two study treatment visits separated by an intervisit interval of 3-14 days. During each study treatment visit, subjects self-administered 10 inhalations within 5 minutes of one of the randomized treatments: epinephrine HFA MDI (Epi-HFA, $125 \mu \mathrm{g} /$ inhalation, total dose $1250 \mu \mathrm{g}$ ) and epinephrine CFC MDI (Epi-CFC, $220 \mu \mathrm{g} /$ inhalation, total dose $2200 \mu \mathrm{g}$ ). Subjects were trained at screening and each study visit for correct dosing.

The study was conducted in accordance with Good Clinical Practice, including the International Conference on Harmonization Guidelines and the Declaration of Helsinki of the World Medical Association. The study protocol and informed consent form was approved by an institutional review board, and all subjects provided written informed consent before screening. The study was registered on the ClinicalTrials.gov (identifier NCT01188577).

\section{Study population}

To allow a conservative evaluation, a sample size of 18 subjects was required. The study population comprised healthy men and nonpregnant women 18-30 years of age with a body mass index of $18.5-30.0 \mathrm{~kg} / \mathrm{m}^{2}$, body weight $\geq 45 \mathrm{~kg}$ for women, and $\geq 50 \mathrm{~kg}$ for men, and sitting blood pressure $\leq 135 /$ $90 \mathrm{mmHg}$. Subjects demonstrated negative alcohol/drug screen tests, $\mathrm{HIV}, \mathrm{HBsAg}$, and $\mathrm{HCV}-\mathrm{Ab}$ screen tests.

Subjects were ineligible if they had a smoking history of $\geq 10$ pack-years, or lower respiratory tract infection within 4 weeks before screening. Subjects also were excluded if they had respiratory conditions, clinically significant cardiovascular and other systemic or organic illnesses which, per investigator discretion, may impact the subjects and/or the study. Other exclusions included use of prohibited medications, or intolerance to any of the study ingredients. Subjects could be discontinued from the study early at the discretion of the investigator owing to medical safety, noncompliance, or administrative concerns.

\section{PK assessments}

Blood samples were collected at predose baseline (0 minutes) and at $2,5,7.5,10,12.5,15,20,25,30$, and 45 minutes, and at 1, 1.5, 2, 4, and 6 hours postdose to construct the plasma concentration-time curve for PK analysis of epinephrine HFA-d3 (1250 $\mu \mathrm{g})$ and epinephrine CFC $(2200 \mu \mathrm{g})$, which served as active control.

At each PK sampling point, blood samples $(\sim 10 \mathrm{~mL})$ were collected in ice-chilled potassium-ethylenediaminetetraacetic acid sample tubes, each containing preadded $1 \%(\mathrm{~V}: \mathrm{V}) 1.0 \mathrm{M}$ sodium metabisulfite solution as an antioxidant. The sample tubes were well mixed immediately, kept on ice or refrigerated, and centrifuged within 60 minutes of collection. The harvested plasma from each sample were aliquoted to two storage tubes $(\sim 2-2.5 \mathrm{~mL} /$ tube $)$, and stored frozen at less than or equal to $-20^{\circ} \mathrm{C}$ until analysis. These samples were tested by a validated liquid chromatography/mass spectrometry/mass spectrometry method with a calibration range of $20-2500 \mathrm{pg} / \mathrm{mL}$ and quantitative limit of $20 \mathrm{pg} / \mathrm{mL}$ (ppt). Plasma concentrations of epinephrine-d3 (exogenous) and epinephrine-h3 (endogenous) were analyzed for Epi-HFA.

For each PK concentration-time curve, including real (exogenous epinephrine) and apparent (total epinephrine) PK curves, the following parameters were obtained: the area under the plasma drug concentration-time curve (AUC) from time 0 (predose) until the last measurable drug concentration (area under the curve, $\mathrm{AUC}_{0-t}$ ), peak concentration from the PK curve $\left(C_{\max }\right)$, and time corresponding to $C_{\max }\left(t_{\max }\right)$.

The primary PK endpoint was to compare the systemic exposure of Epi-HFA and Epi-CFC based on $\mathrm{AUC}_{0-t}$ of the apparent (total epinephrine) PK curves of Epi-HFA and that of Epi-CFC. The secondary endpoints included $C_{\max }, t_{\max }$, and relative bioavailability (RBA) between Epi-HFA versus Epi-CFC.

The plasma epinephrine data at normal doses was calculated based on the following formula:

$$
C_{\text {Total }}^{\text {Normal }}(t)=\frac{C_{\text {Total }}^{\mathrm{High}}(t)-b}{5}+b
$$

where $1 / 5$ denotes the fivefold ratio between high dose (10 puffs) and normal dose ( 2 puffs) and $b$ denotes the endogenous epinephrine levels (based on average endogenous levels during the first hour after dosing).

\section{Safety measurements}

Safety assessments included monitoring of AEs, vital signs including systolic blood pressure (SBP), diastolic blood pressure (DBP), and heart rate (HR), 12-lead electrocardiogram (ECG) (routine and QT/QTc intervals), clinical laboratory testing, and physical examinations were evaluated. AEs were coded using the Medical Dictionary for Regulatory Activities terminology.

\section{Statistical analysis}

The primary analysis was to evaluate PK profile of EpiHFA that includes the concentration-time curve and PK parameters such as AUC and $C_{\max }$. The main parameters were analyzed in all randomized subjects who received at 
least one dose of study drug treatment and had sufficient PK measurements. Pairwise comparisons of $\mathrm{AUC}_{0-t}$ between treatments were performed using one-sided $t$-tests with $\alpha=0.05$. Epi-HFA is comparable or has a significantly less systemic drug exposure than that for Epi-CFC if $p \leq 0.05$. Similar statistical analysis was performed for other PK parameters including $C_{\max }$. Both real and apparent PK curves were fitted with the most suitable PK model by the standard PK analysis software Phoenix ${ }^{\circledR}$ WinNonlin (Certara USA, Inc., Princeton, NJ).

Safety parameters were assessed in all randomized subjects who received at least one dose of study drug treatment.

\section{Results}

\section{Subject demographics}

A total of 35 subjects were screened and 23 (65.7\%) were randomized to receive study treatment (Table 1). All the randomized subjects $(100 \%)$ completed the study and were included in the PK and safety analyses. The majority of subjects were African American (60.9\%) and men (73.9\%) with the mean age of 24 years (Table 2).

\section{PKs assessment}

The plasma epinephrine levels for both exogenous epinephrine (epinephrine hfa-d3) and endogenous epinephrine for Epi-HFA at high dose are given in Table 3. For the active control, Epi-CFC, only total epinephrine (both exogenous and endogenous) was tested. Based on the highdose experimental data, the plasma epinephrine levels at normal dose for Epi-HFA and Epi-CFC were extrapolated and the results are given in Table 3 and Figure 1.

For total epinephrine, Epi-HFA demonstrated a greater systemic drug exposure (AUC) than that of Epi-CFC. The AUC for total epinephrine evaluated by statistical analysis was $8.5 \pm 5.2$ for Epi-HFA and $6.2 \pm 4.1 \mathrm{ng} /(\mathrm{mL} \cdot \mathrm{min})$ for Epi-CFC. Thus, the RBA of Epi-HFA was calculated to be $137 \%$, which indicates that the AUC of Epi-HFA $(1250 \mu \mathrm{g}$ inhaled) was $37 \%$ greater than that of Epi-CFC $(2200 \mu \mathrm{g}$ inhaled) with a $p$-value of 0.052 . Furthermore, the peak concentration $\left(C_{\max }\right)$ in the blood system occurred at 2 minutes for Epi-HFA and was statistically greater (4.5 times) than Epi-CFC, with $p<0.0001$ (Table 3). During this time, the plasma epinephrine levels that would result after a

\section{Table 1. Subjects Disposition}

\begin{tabular}{lcc}
\hline No. of subjects screened & 35 & \\
$\begin{array}{l}\text { No. of subjects } \\
\text { randomized }\end{array}$ & 23 & \\
& & Epinephrine \\
HFA-d3 1.25 mg & $\begin{array}{c}\text { Epinephrine } \\
\text { CFC 2.20 mg }\end{array}$ \\
$\begin{array}{c}\text { No. of subjects received } \\
\text { study treatment(s) }\end{array}$ & 23 & 23 \\
$\begin{array}{l}\text { No. of subjects completed } \\
\text { study period with }\end{array}$ & 23 & 23 \\
$\begin{array}{l}\text { PK data available } \\
\text { No. of subjects completed } \\
\text { study for crossover } \\
\text { analysis vs. control }\end{array}$ & 23 & \\
\hline
\end{tabular}

PK, pharmacokinetic; HFA, hydrofluoroalkane; CFC, chlorofluorocarbon.
Table 2. Demographic Data

\begin{tabular}{lc}
\hline Subject characteristics & Crossover $(\mathrm{n}=23)$ \\
\hline Age (years) & \\
Mean \pm SD & $23.8 \pm 3.1$ \\
Range & $18-29$ \\
Gender, $\%$ & \\
Male & 73.9 \\
Female & 26.1 \\
Weight (kg) & \\
Mean \pm SD & $72.0 \pm 11.8$ \\
Range & $54-92$ \\
Height (cm) & \\
Mean \pm SD & $172.5 \pm 10.6$ \\
Range & $153-194$ \\
Race group, \% & \\
Caucasian & 30.4 \\
African American & 60.9 \\
Asian & 8.7 \\
Others & 0.0 \\
\hline
\end{tabular}

$\mathrm{SD}$, standard deviation.

"normal" maximal dose of two puffs were calculated to be $180 \mathrm{pg} / \mathrm{mL}$ for Epi-HFA and $46 \mathrm{pg} / \mathrm{mL}$ for Epi-CFC. By 10 minutes after the $t_{\max }$ (at $\sim 12.5$ minutes postdose), the plasma epinephrine level was reduced to about one-tenth of the $C_{\max }$ showing an elimination half-life $\left(t_{1 / 2}\right)$ of 2.6 minutes. The duration of higher plasma levels seen with Epi-HFA was brief (5-10 minutes), and plasma levels were found to be comparable with Epi-CFC by 20 minutes postdose and for all subsequent time points out to 360 minutes (Table 3). Within 60 minutes after inhalation, the radiolabeled exogenous epinephrine concentrations in plasma declined to an undetectable level in the blood plasma. The extrapolated PK curves for normal (two puff) doses of both Epi-HFA and Epi-CFC well matched a one-compartment model with the first-order input and first-order output (Fig. 1).

\section{Safety assessment}

The overall extent of the study drug exposure for each subject was $1.25 \mathrm{mg}$ of Epi-HFA and $2.20 \mathrm{mg}$ of Epi-CFC delivered as single 10-puff doses on two separate treatment days. After completion of the crossover study visits, the total exposure of epinephrine inhaler was $3.45 \mathrm{mg}$.

A total of three AEs were reported, of which one (diarrhoea) was for Epi-HFA and two (cough and viral upper respiratory tract infection) for Epi-CFC. All AEs were classified as mild and not serious by the investigators. All were resolved without residual effects. The clinical laboratory data were within the normal range.

No significant effect to vital signs and ECG variables were found comparing high doses of Epi-HFA and EpiCFC. The changes in vital signs and ECG were comparable with no substantial differences observed between the two study drug treatments. Both formulations of epinephrine did produce characteristic cardiovascular changes, with moderate increases in SBP and DBP and HR at 30 minutes postdose. Epi-HFA had a slightly higher increase in SBP and DBP (Table 4), and by 60 minutes, a normalization in both SBP and DBP was observed. There were two single cases of premature ventricular contractions (PVCs) in the Epi-HFA 
Table 3. Plasma Epinephrine Concentration After High-Dose Inhalation and Normalized Dose

\begin{tabular}{|c|c|c|c|c|c|c|}
\hline \multirow{2}{*}{$\begin{array}{l}\text { Time after } \\
\text { inhalation } \\
\text { (minutes) }\end{array}$} & \multicolumn{4}{|c|}{ Experimental data at high dose $(\mathrm{ng} / \mathrm{mL})$} & \multicolumn{2}{|c|}{$\begin{array}{l}\text { Calculated data at } \\
\text { normal dose }(\mathrm{ng} / \mathrm{mL})\end{array}$} \\
\hline & $\begin{array}{l}\text { Exogenous } \\
\text { Epi-HFA-d3 }\end{array}$ & $\begin{array}{l}\text { Endogenous } \\
\text { Epi-HFA-h3 }\end{array}$ & $\begin{array}{l}\text { Total Epi-HFA } \\
10 \times 125 \mu \mathrm{g}\end{array}$ & $\begin{array}{c}\text { Total Epi-CFC } \\
10 \times 220 \mu \mathrm{g}\end{array}$ & $\begin{array}{l}\text { Epi-HFA } \\
2 \times 125 \mu \mathrm{g}\end{array}$ & $\begin{array}{l}\text { Epi-CFC } \\
2 \times 220 \mu g\end{array}$ \\
\hline 0 & 0 & 3 & 2.6 & 4.3 & 9 & 9 \\
\hline 2 & 856 & 6 & 862 & 189 & 180 & 46 \\
\hline 5 & 378 & 2 & 379 & 99 & 84 & 28 \\
\hline 7.5 & 184 & 3 & 187 & 49 & 45 & 18 \\
\hline 10 & 111 & 7 & 118 & 40 & 32 & 16 \\
\hline 12.5 & 71 & 4 & 76 & 34 & 23 & 15 \\
\hline 15 & 39 & 8 & 47 & 32 & 17 & 14 \\
\hline 20 & 20 & 3 & 23 & 30 & 13 & 14 \\
\hline 25 & 8 & 3 & 11 & 20 & 10 & 12 \\
\hline 30 & 6 & 5 & 11 & 18 & 10 & 12 \\
\hline 45 & 2 & 8 & 11 & 20 & 10 & 12 \\
\hline 60 & 1 & 8 & 9 & 13 & 10 & 11 \\
\hline 90 & 0 & 4 & 4 & 11 & 9 & 10 \\
\hline 120 & 0 & 7 & 7 & 7 & 9 & 9 \\
\hline 240 & 0 & 16 & 16 & 18 & 11 & 12 \\
\hline 360 & 0 & 17 & 17 & 17 & 11 & 11 \\
\hline
\end{tabular}

group that occurred with no arrhythmias. The PVC events occurred at time point of 6 hours postdose and were considered not clinically significant.

\section{Discussion}

With respect to PKs, the overall systemic exposure of epinephrine HFA was found to be low. The $C_{\max }$ in both
Epi-HFA and Epi-CFC occurred at 2 minutes $\left(t_{\max }\right)$ postdose and was higher in Epi-HFA $(180 \mathrm{pg} / \mathrm{mL})$ at normal dose compared with that for Epi-CFC $(46 \mathrm{pg} / \mathrm{mL})$. Although plasma epinephrine levels were relatively higher in the EpiHFA group, this was found to be a transient effect occurring in the first few minutes after drug administration. Systemic epinephrine levels decreased rapidly with a half-life of 2.6 minutes and were comparable between the two study drugs

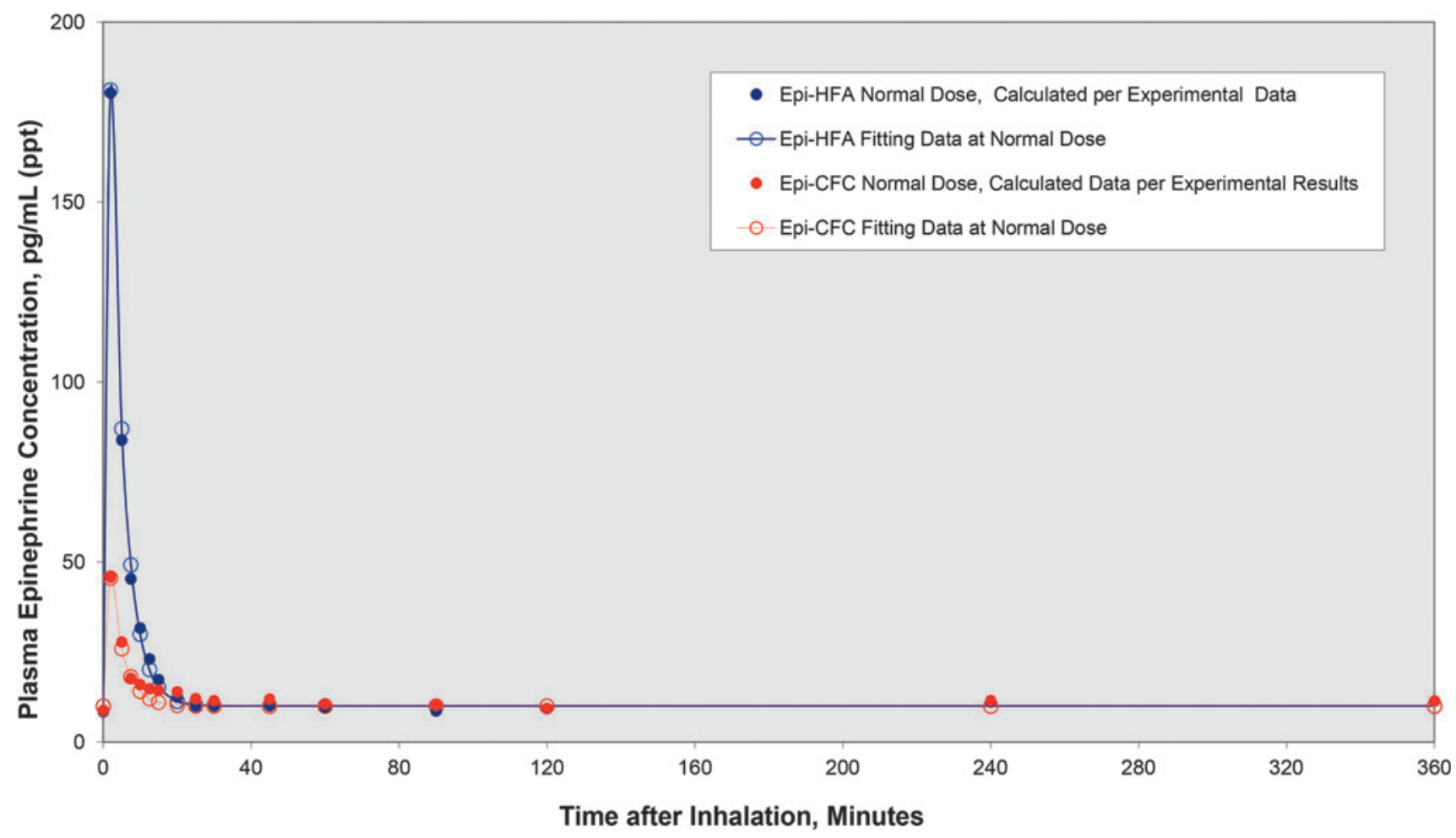

FIG. 1. Extrapolated plasma epinephrine concentration curves at normal dose: Peak plasma epinephrine concentration levels occurred at 2 minutes postdose, $180 \mathrm{pg} / \mathrm{mL}$ for Epi-HFA and $46 \mathrm{pg} / \mathrm{mL}$ for Epi-CFC, respectively. HFA, hydrofluoroalkane; CFC, chlorofluorocarbon. 
Table 4. Vital Signs and Electrocardiogram for High Dose of Study Drug Treatments

\begin{tabular}{|c|c|c|c|c|c|c|c|c|c|}
\hline \multirow[b]{2}{*}{ Items } & \multirow[b]{2}{*}{$\begin{array}{c}\text { Time after } \\
\text { drug inhaled } \\
\text { (minutes) }\end{array}$} & \multicolumn{4}{|c|}{ Epi-HFA-d3 $1.25 \mathrm{mg}, \mathrm{n}=23$} & \multicolumn{4}{|c|}{ Epi-CFC $2.20 \mathrm{mg}, \mathrm{n}=23$} \\
\hline & & $\begin{array}{c}\text { Mean of } \\
\text { data }\end{array}$ & $\begin{array}{c}\text { Mean } \\
\text { of } \Delta\end{array}$ & $\begin{array}{c}\text { Upper } \\
95 \% \\
\text { CI of } \Delta\end{array}$ & $\begin{array}{c}\text { Mean } \\
\Delta \%\end{array}$ & $\begin{array}{c}\text { Mean of } \\
\text { data }\end{array}$ & $\begin{array}{c}\text { Mean } \\
\text { of } \Delta\end{array}$ & $\begin{array}{l}\text { Upper } \\
95 \% C I \\
\text { of } \Delta\end{array}$ & $\begin{array}{c}\text { Mean } \\
\Delta \%\end{array}$ \\
\hline \multirow[t]{4}{*}{$\mathrm{SBP}, \mathrm{mmHg}$} & Baseline & 115 & - & - & - & 116 & - & - & - \\
\hline & 30 & 125 & 10.1 & 13.5 & 9.2 & 122 & 6.6 & 9.6 & 6.1 \\
\hline & 60 & 115 & -0.5 & 3.1 & 0.1 & 113 & -2.7 & 1.8 & -1.6 \\
\hline & 360 & 122 & 6.8 & 10.2 & 6.2 & 121 & 5.2 & 7.0 & 4.7 \\
\hline \multirow[t]{4}{*}{$\mathrm{DBP}, \mathrm{mmHg}$} & Baseline & 60 & - & - & - & 60 & - & - & - \\
\hline & 30 & 64 & 4.5 & 7.5 & 8.3 & 63 & 2.6 & 5.0 & 4.4 \\
\hline & 60 & 62 & 2.7 & 6.9 & 5.8 & 59 & -1.4 & 1.0 & -1.9 \\
\hline & 360 & 61 & 1.8 & 4.4 & 4.2 & 62 & 2.1 & 3.7 & 3.8 \\
\hline \multirow{4}{*}{$\begin{array}{l}\text { HR, bpm } \\
\quad \text { (by vital sign) }\end{array}$} & Baseline & 61 & - & - & - & 61 & - & - & - \\
\hline & 30 & 65 & 4.3 & 6.0 & 7.0 & 65 & 4.2 & 6.7 & 7.4 \\
\hline & 60 & 63 & 2.1 & 3.9 & 3.4 & 61 & 0.0 & 2.0 & 0.1 \\
\hline & 360 & 65 & 3.9 & 5.7 & 6.7 & 66 & 5.6 & 6.9 & 9.4 \\
\hline \multirow{3}{*}{$\begin{array}{l}\mathrm{HR} \text {, bpm } \\
\text { (by ECG) }\end{array}$} & Baseline & 60 & - & - & - & 61 & - & - & - \\
\hline & 30 & 64 & 3.3 & 5.1 & 5.6 & 64 & 3.2 & 5.5 & 6.1 \\
\hline & 360 & 67 & 6.6 & 9.1 & 11.4 & 65 & 4.7 & 6.7 & 7.8 \\
\hline \multirow[t]{3}{*}{$\mathrm{QT}, \mathrm{ms}$} & Baseline & 406 & - & - & - & 404 & - & - & - \\
\hline & 30 & 397 & -9.5 & -1.4 & -2.2 & 395 & -8.9 & -4.1 & -2.1 \\
\hline & 360 & 387 & -19.0 & -10.0 & -4.5 & 391 & -13.0 & -6.7 & -3.3 \\
\hline \multirow[t]{3}{*}{ QTc, ms } & Baseline & 402 & - & - & - & 401 & - & - & - \\
\hline & 30 & 403 & 1.4 & 5.8 & 0.4 & 404 & 2.3 & 7.9 & 0.6 \\
\hline & 360 & 403 & 1.7 & 6.3 & 0.5 & 402 & 0.7 & 5.5 & 0.2 \\
\hline \multirow[t]{3}{*}{ QTc-B, ms } & Baseline & 403 & - & - & - & 402 & - & - & - \\
\hline & 30 & 404 & 1.2 & 5.6 & 0.3 & 405 & 2.4 & 8.0 & 0.6 \\
\hline & 360 & 404 & 1.5 & 6.2 & 0.4 & 403 & 0.8 & 5.7 & 0.2 \\
\hline \multirow[t]{3}{*}{ QTc-F, ms } & Baseline & 404 & - & - & - & 402 & - & - & - \\
\hline & 30 & 401 & -2.4 & 2.6 & -0.5 & 401 & -1.3 & 2.6 & -0.3 \\
\hline & 360 & 398 & -5.5 & -0.6 & -1.3 & 398 & -4.0 & 0.5 & -1.0 \\
\hline
\end{tabular}

SBP, systolic blood pressure; DBP, diastolic blood pressure; HR, heart rate; CI, confidence interval; ECG, electrocardiogram.

by 20 minutes postdose. Despite the higher $C_{\max }$, minimal adverse effects were observed in Epi-HFA even after administration of very high doses (10 inhalations).

One possible explanation for the low occurrence of adverse effects is that when considering that the typical blood volume for an adult is 4.7-5 L, the amount of epinephrine distributed into the adult blood system from Epi-HFA at peak $C_{\max }(180 \mathrm{pg} / \mathrm{mL})$ would only be $0.36 \%(0.9 \mu \mathrm{g} / 250$ $\mu \mathrm{g})$ of the total amount of epinephrine inhaled. This suggests that most of the epinephrine may be retained and locally metabolized in the patients' lungs, or it may be absorbed over several hours. Furthermore, this amount $(180 \mathrm{pg} / \mathrm{mL})$ does not appear to be a safety concern, as it represents only $\sim 20 \%$ of the normal human endogenous epinephrine levels measurable during vigorous exercise,* $5 \mathrm{nmol} / \mathrm{L}$ or $0.92 \mathrm{ng} / \mathrm{mL}^{(5,6)}$

The bioavailability and cardiovascular results found in this study were consistent with past publications on the inhalation of epinephrine, which did not find significant safety risks with epinephrine MDI even at high dosages. ${ }^{(7,8)}$ In the previous study of epinephrine HFA at high dose (i.e., 10 inhalations), an increase in systolic and diastolic blood

*Exercise level is defined as $110 \%$ max volume of oxygen $\left(\mathrm{VO}_{2} \mathrm{max}\right)$, that is, when running 2 minutes at the speed of $9.7 \mathrm{~km} / \mathrm{h}$ for $323 \mathrm{~m}$, the epinephrine level is in the range of $0.67-1.6 \mathrm{ng} / \mathrm{mL}$. ${ }^{6}$ pressure and HR were noted at 10 minutes after dosing that returned to baseline by 360 minutes. ${ }^{(9)}$ Similar to the effects seen in this study, these changes in vital signs are associated with the use of epinephrine HFA beyond the labeled dose, which was taking 10 inhalations of $125 \mu \mathrm{g}$ in rapid succession. The changes in blood pressure and HR are expected to be minimal with the labeled dose.

Despite the common concern regarding the cardiovascular effects of epinephrine, ${ }^{(3)}$ data on the serious side effects of epinephrine metered-dose inhalation in the literature are scarce. Serious effects were observed when epinephrine was given by injection where systemic bioavailability of the drug approaches $100 \% .^{(10,11)}$ A safety review on epinephrine found that the most serious cardiac adverse effects occur when epinephrine is given by intravenous injection (e.g., severe myocardial ischemia). ${ }^{(1)}$ Therefore, a future study exploring the PK safety between epinephrine MDIs and epinephrine injection will be worthwhile.

A potential limitation of this study is that it was conducted in healthy subjects instead of asthmatic patients in whom the drug will be used. Furthermore, the study design was restricted to subjects at the ages of 18-30. Therefore, further studies evaluating subjects at a wider age range would provide a broader PK analysis.

In conclusion, the systemic exposure of epinephrine HFA was found to be low in this PK study, with levels similar to epinephrine CFC after the initial 10-20 minutes postdose. 
There was an increased cardio-dynamic effect noted at 30 minutes postdose with both high-dose inhaled formulations, but few AEs and no clinically important safety findings were reported in this study.

\section{Author Disclosure Statement}

Dr. E.M.K. served on advisory boards, speaker panels, consultants, or received travel reimbursement from Novartis, AstraZeneca, Amphastar, Forest, Pearl, Sunovion, Teva, Theravance, Mylan, GSK, Boehringer Ingelheim, and Cipla outside the submitted work. Dr. J.Y.Z., Dr. M.Z.L. and T.M. are employees of Amphastar Pharmaceuticals, Inc. at the time of study and article preparation.

\section{Funding Information}

Amphastar Pharmaceuticals, Inc. funded the pharmacokinetic study and the preparation of the article, including all article processing charges. The two study drugs, epinephrine HFA MDI (Primatene ${ }^{\circledR}$ MIST) and epinephrine CFC MDI (Primatene $^{\circledR}$ MIST $^{\circledR}$ CFC) were provided by Armstrong Pharmaceuticals, Inc., a subsidiary of Amphastar Pharmaceuticals, Inc.

\section{References}

1. Federal Register Volume 73, no 224: 21 CFR part 2: Use of ozone-depleting substances; removal of essential-use designation (Epinephrine) final rule. November 19, 2008.

2. Gao J, Luo J, Zhou R, Luo MZ, and Zhang JY: Stable epinephrine suspension formulation with high inhalation delivery efficiency. US Patent 8,367,734 B1. February 5, 2013. Available from: https://patentimages.storage.googleapis .com/67/65/d4/461c6f9a24c31a/US8367734.pdf (Accessed on December 18, 2019).

3. National Asthma Education and Prevention Program, Third Expert Panel on the Diagnosis and Management of Asthma. Expert Panel Report 3: Guidelines for the Diagnosis and Management of Asthma. Bethesda (MD): National Heart, Lung, and Blood Institute (US); 2007 Aug. Report No.:074051. Available from: https://www.ncbi.nlm.nih.gov/books/ NBK72321/ [Last accessed September 9, 2015].

4. Thakkar K, Mhatre S, Jadhav M, Goswami S, and Shah R: Pharmacokinetic studies for proving bioequivalence of or- ally inhaled drug products-critical issues and concepts. Front Pharmacol. 2015;6:117.

5. Katzung B: Basic and Clinical Pharmacology. 9th ed. 2004. New York: Lange Medical Books/McGraw/Hill.

6. Kjaer M, Farrell PA, Christensen NJ, and Galbo H: Increased epinephrine response and inaccurate glucoregulation in exercising athletes. J Appl Physiol. 1986;61:1693-1700.

7. Warren JB, Doble N, Dalton N, and Ewan PW: Systemic absorption of inhaled epinephrine. Clin Pharmacol Ther. 1986;40:673-678.

8. Hendeles L, Marshik PL, Ahrens R, Kifle Y, and Shuster J: Response to nonprescription epinephrine inhaler during nocturnal asthma. Ann Allergy Asthma Immunol. 2005;95: 530-534.

9. FDA Briefing Document for a Joint Meeting of the Nonprescription Drugs and the Pulmonary and Allergy Drugs Advisory Committees. Risk/benefit considerations for use of epinephrine as an OTC asthma therapy. Silver Spring, MD, February 25, 2014; pp. 51-52. Available from: http:// wayback.archive-it.org/7993/20170113233021/http://www .fda.gov/downloads/AdvisoryCommittees/CommitteesMeeting Materials/Drugs/NonprescriptionDrugsAdvisoryCommittee/ UCM386675.pdf (Accessed on January 28, 2019).

10. Simons FE, Gu X, and Simons KJ: Epinephrine absorption in adults: Intramuscular versus subcutaneous injection. J Allergy Clin Immunol. 2001;108:871-873.

11. Wood JP, Traub SJ, and Lipinski C: Safety of epinephrine for anaphylaxis in the emergency setting. World J Emerg Med. 2013;4:245-251.

Received on October 15, 2019 in final form, April 7, 2020

Reviewed by: Tunde Otulana Gur Jai Pal Singh

Jack Y. Zhang, PhD Amphastar Pharmaceuticals, Inc. 11570 6th Street Rancho Cucamonga, CA 91730

USA

E-mail: executiveoffice@ims-limited.com 